\title{
The role of genetic serotonin transporter deficiency in consequences of exposure to the Western diet: a study in mice
}

\begin{abstract}
U. Ekaterina Veniaminova ${ }^{1,2}$, Anna Gorlova $^{1,2}$, Jenna Hebert ${ }^{3}$, Fay Probert ${ }^{3}$, Daniel Radford-Smith ${ }^{3}$, Raymond Cespuglio ${ }^{1,4}$, Angelika G. Schmitt-Boehrer5; Klaus-Peter Lesch ${ }^{1,2,5}$, Daniel C. Anthony ${ }^{3}$, Tatyana Strekalova ${ }^{1,2}$

1'Laboratory of Psychiatric Neurobiology, Institute of Molecular Medicine, Sechenov First Moscow State Medical University, Russia; 2Department of Neuroscience, School for Mental Health and Neuroscience, Maastricht University, Netherlands; ${ }^{3}$ Department of Pharmacology, Oxford University, United Kingdom; ${ }^{4}$ Claude Bernard University Lyon 1, France; ${ }^{5}$ Division of Molecular Psychiatry, Laboratory of Translational Neuroscience, Center of Mental Health, University of Würzburg, Germany
\end{abstract}

\section{Introduction}

The "Western diet" (WD), a diet enriched with fat and cholesterol, is associated with obesity and development of metabolic syndrome and type 2 diabetes and increased incidence of affective disorders ${ }^{1}$. Carriers of $S$ allele of serotonin transporter (SERT), a genetic variant that reduces SERT expression, are more susceptible to emotionality-related disorders ${ }^{2}$ and prone to obesity and diabetes ${ }^{3}$.

Aim: investigate the interaction of SERT deficit and WD consumption in aging with regard to their effects on behavioural, metabolic and molecular parameters using a mouse model.

\section{Study design}

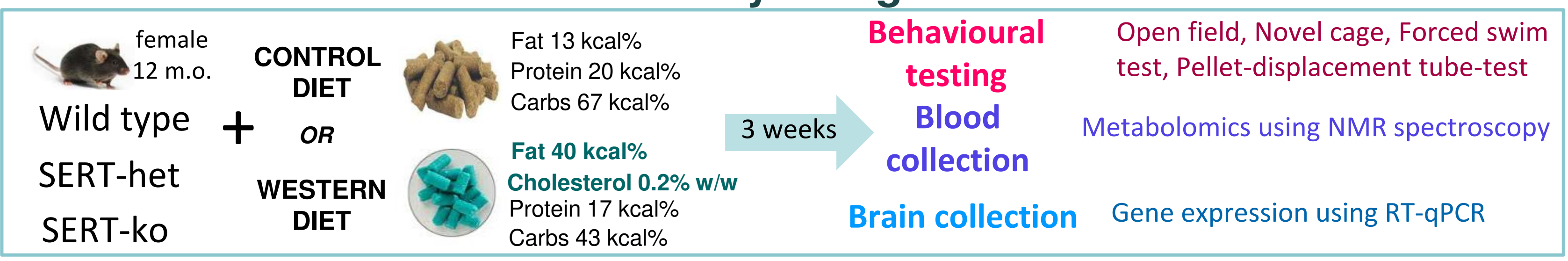

\section{Results}

3.1 Increased depressive-like behaviour and altered cognitive functions in SERT-ko mice fed WD

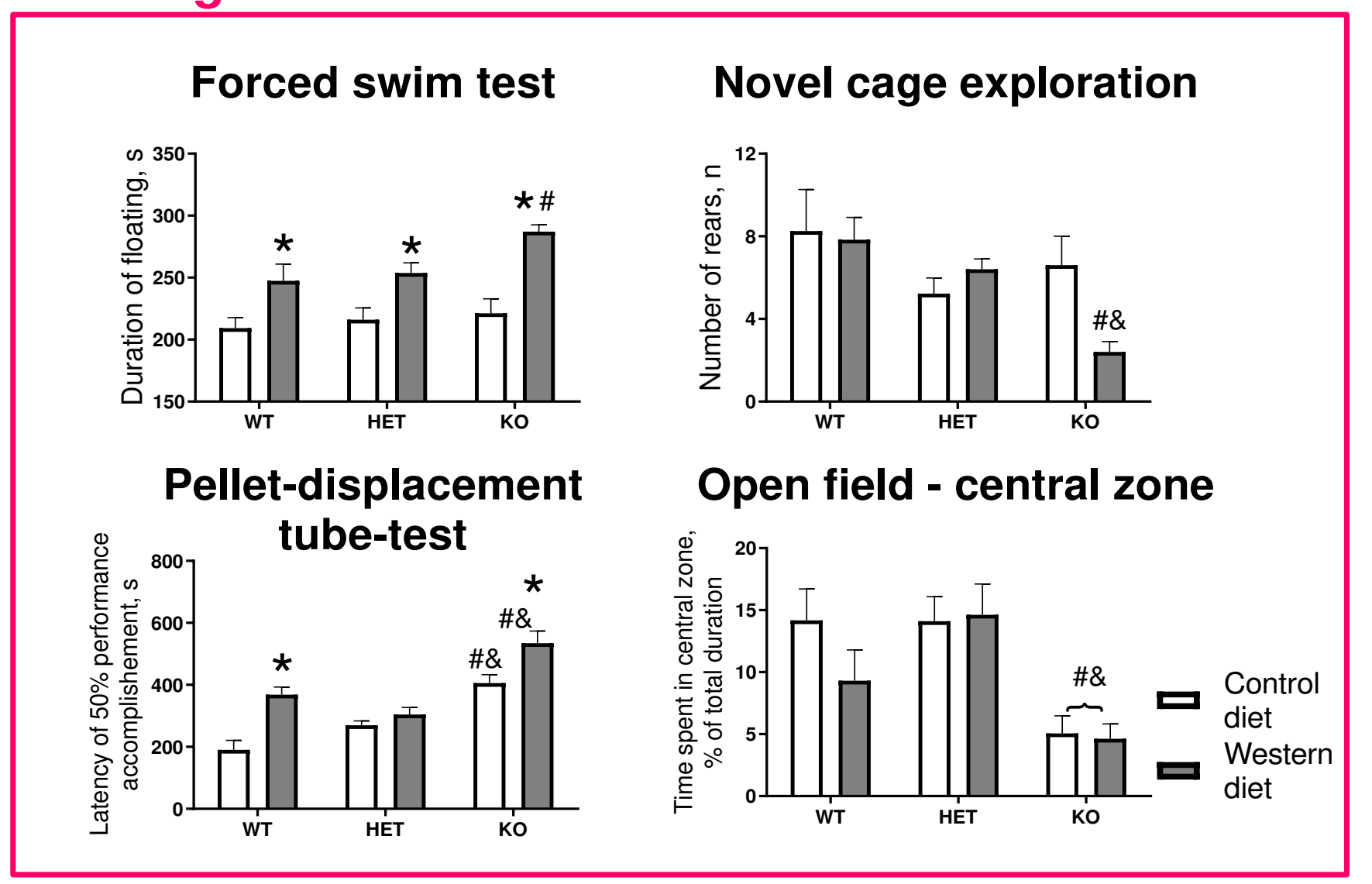

3.3 Altered gene expression in hypothalamus of SERT-KO mice

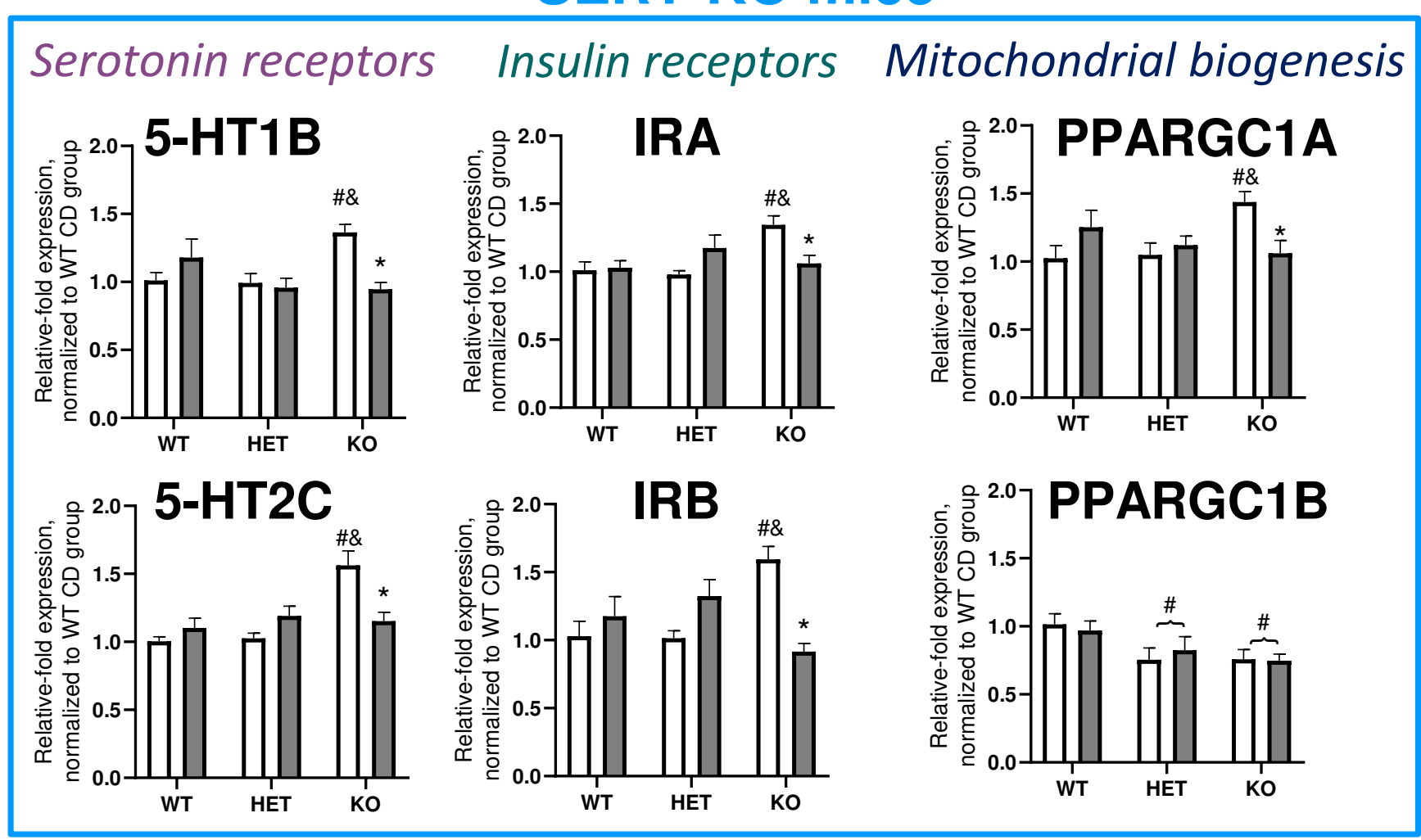

3.2 Genetic absence of SERT exacerbates changes in blood metabolome induced by WD

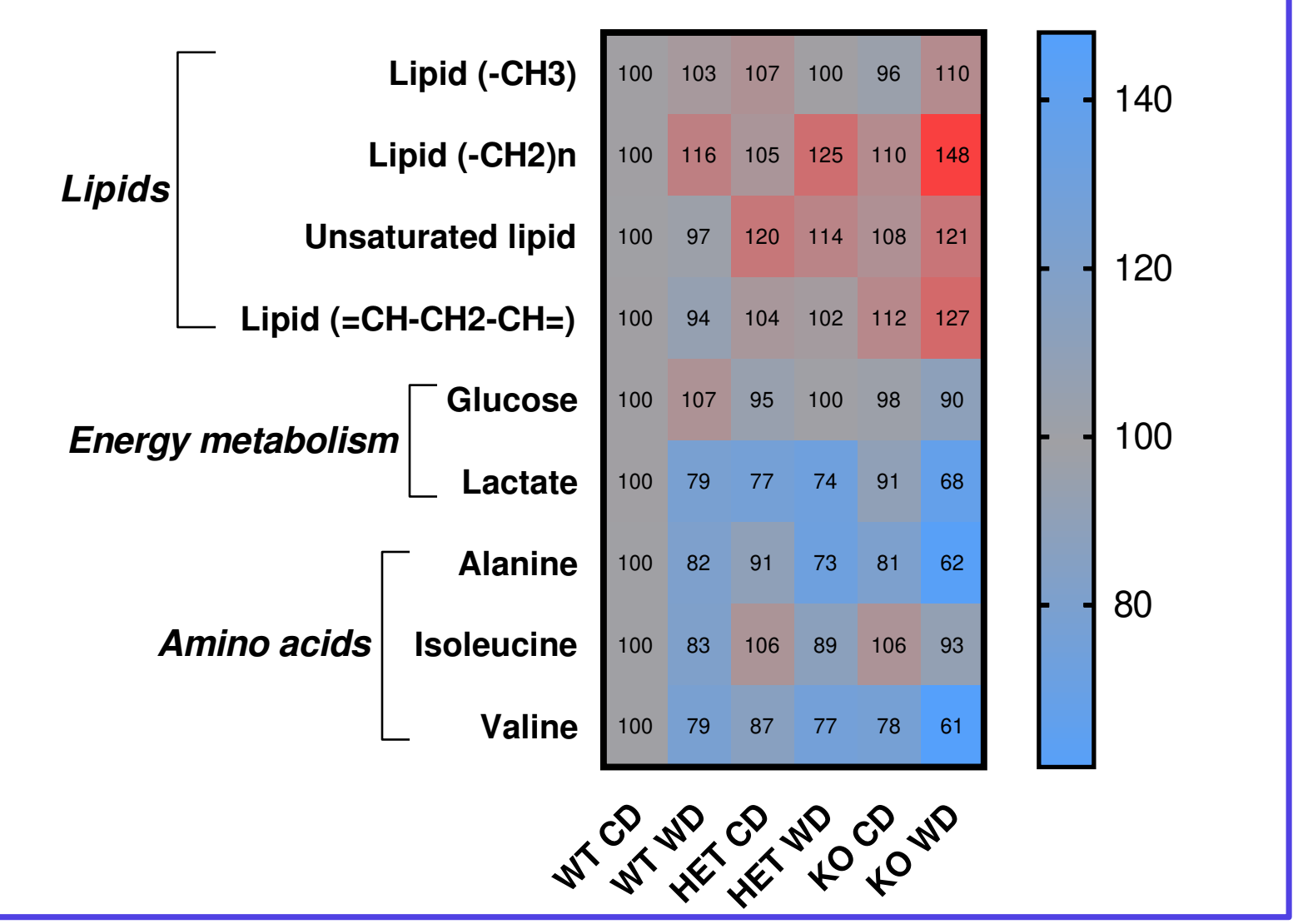

\section{Research highlights}

1. Changes in metabolome, depressive-like behaviour and cognitive dysfunctions induced by Western diet are exacerbated in SERT-ko mice

2. Increased expression of serotonin and Insulin receptors and factor of mitochondrial biogenesis PPARGC1A in control SERT-ko mice may reflect compensatory CNS changes caused by serotoninergic system dysregulation in these animals. Western diet inverses compensatory changes in serotonin and insulin receptors and PPARGC1A expression in SERT-ko mice

3. SERT-het mice show resilience to the effects of Western diet on behaviour in tube-test 